\title{
Real world clinical outcomes of adjuvant sequential chemoradiation in patients with gallbladder carcinomas with poor performance status
}

\author{
Rakesh Kapoor ${ }^{1,2}$, Kannan Periasamy ${ }^{2}$, Rajesh Gupta ${ }^{3}$, Arun Yadav², Divya Khosla ${ }^{2}$ \\ ${ }^{1}$ Department of Radiotherapy, Homi Bhabha Cancer Hospital and Research Center, Mullanpur \& Sangrur, India \\ ${ }^{2}$ Department of Radiotherapy and Oncology, Postgraduate Institute of Medical Education and Research (PGIMER), Chandigarh, India \\ ${ }^{3}$ Department of Surgical Gastroenterology, Postgraduate Institute of Medical Education and Research (PGIMER), Chandigarh, India
}

Received: August 30, 2020

Revised: December 10, 2020

Accepted: December 14, 2020

Correspondence:

Kannan Periasamy

Department of Radiotherapy and

Oncology, Postgraduate Institute of

Medical Education and Research

(PGIMER), Chandigarh 160012, India

Tel: +91-9442395508

E-mail: pkannan10011988@gmail.com ORCID

https://orcid.org/0000-0001-7440-0996
Purpose: The purpose of the study is to analyze the overall survival, relapse-free survival, and relapse patterns of adjuvant sequential chemoradiation for gallbladder cancers after curative resection in patients with poor performance status.

Materials and Methods: We retrospectively reviewed clinical records of gallbladder patients with pathologic stage T2-4 or node positivity treated with sequential chemoradiation at our institute between January 2015 and January 2019. Sequential chemoradiotherapy protocol consisted of six cycles of gemcitabine $1,000 \mathrm{mg} / \mathrm{m}^{2}$ and oxaliplatin $100 \mathrm{mg} / \mathrm{m}^{2}$ administered every 2 weekly and postoperative radiation therapy ( $45 \mathrm{~Gy}$ in 25 fractions over 5 weeks) by three-dimensional conformal technique.

Results: A total of 36 patients were included. The median overall survival and relapse-free survival was 26 months (95\% confidence interval [Cl], 21.4-30.5) and 21 months (95\% Cl, 11.8-30.1), respectively. The 2-year overall and relapse-free survival rates were $55.1 \%(95 \% \mathrm{Cl}, 37.9 \%-72.3 \%)$ and $44.7 \%$ (95\% Cl, 27.5\%-61.9\%), respectively. Locoregional, systemic, and combined recurrence were noted in 2 (5.5\%), 14 (38.8\%), and $3(8.3 \%)$ patients, respectively. On univariate analysis, tumour grading significantly influenced relapse free survival; nodal stage and overall stage demonstrated a statistically significant influence on overall survival $(p<0.05)$ with a trend towards significance for lymphovascular invasion. On multivariate analysis, no significant factors were found. Grade 3 and 4 haematological adverse events were observed only in 2 (5.5\%) with chemotherapy. No grade 3 and 4 adverse events were observed due to radiation therapy.

Conclusion: Sequential chemoradiation is feasible and tolerable with acceptable efficacy in the adjuvant setting in patients unfit for concurrent chemoradiotherapy.

Keywords: Gallbladder neoplasms, Cholecystectomy, Chemoradiotherapy, Three-dimensional conformal radiotherapy, Survival, Recurrence

\section{Introduction}

Biliary tract cancers constitute a heterogeneous group of neoplasms arising from the epithelium of bile ducts. They are classified into cancers of the gallbladder and cholangiocarcinomas, which can be either intrahepatic or extrahepatic [1]. Cancers of the gallbladder account for bulk of biliary tract neoplasms. In India, according to the GLOBOCAN 2018, approximately 25,999 new cases of gallbladder cancer are diagnosed every year and gallbladder cancer ranks 13th among cancer-related mortality. India contrib-

Copyright (C) 2020 The Korean Society for Radiation Oncology

This is an Open Access article distributed under the terms of the Creative Commons Attribution Non-Commercial License (http://creativecommons.org/licenses/by-nc/4.0/) which permits unrestricted non-commercial use, distribution, and reproduction in any medium, provided the original work is properly cited. 
utes to approximately $12 \%$ of the global burden of gallbladder cancer [2-4]. Surgery is the definitive treatment for cure for localized resectable cancers which account for approximately 10\%$20 \%$ of tumours [5-7]. After curative resection alone, the recurrence remains high with the literature review suggesting a locoregional recurrence rate of 30\%-65\% and distant recurrence as high as $80 \%[8,9]$. These findings reveal that relapses, both locoregional and distant, are common after curative resection, rationalizing the consideration of use of radiation and systemic therapy in the adjuvant setting to reduce the relapse rates and thereby improve the survival and quality of life. Various treatment options that have been utilized include surveillance, adjuvant chemotherapy, adjuvant radiotherapy with or without concurrent chemotherapy, and adjuvant chemotherapy followed by concurrent chemoradiotherapy [10-16]. Lacking adequate high quality prospective clinical evidence comparing adjuvant treatment modalities due to low number of resected cases, the benefit of adjuvant therapy in these cancers is often unclear amid several therapeutic options. Sequential chemoradiotherapy consisting of chemotherapy and radiotherapy is the adjuvant therapy followed at our institute after curative resection in patients who are not fit enough to tolerate concurrent chemoradiation. In the present study, we retrospectively analyzed the outcomes of those gallbladder cancers treated with adjuvant sequential chemoradiation.

\section{Materials and Methods}

\section{Study design and patients}

We retrospectively reviewed records of gallbladder cancer patients treated with curative surgery and adjuvant sequential chemoradiation at our institute between January 2015 and January 2019. The flow diagram outlining the selection criteria of the study has been depicted in Fig. 1. Unresectable and metastatic diseases were excluded. Perfectly fit individuals treated with concurrent chemoradiotherapy were also excluded. Sequential chemoradiotherapy is being followed in our institute for patients who are not fit enough to tolerate concurrent chemoradiotherapy-Zubrod performance status 2 or more or with comorbidities. The sequential chemoradiotherapy protocol included 6 cycles of gemcitabine and oxaliplatin and postoperative radiation therapy. Adjuvant therapy was started 4 weeks after surgery. All patients planned for adjuvant sequential chemoradiotherapy were begun treatment with chemotherapy. Radiotherapy was delivered whenever patients obtained their slots in the machine. Eighteen patients received radiation after 2-3 cycles of chemotherapy and 18 patients received radiotherapy after completion of 6 cycles of chemotherapy. A gap of 3-4 weeks was allowed between chemotherapy and radiotherapy. Staging of tu- mours was done retrospectively with the American Joint Committee on Cancer (AJCC) 8th edition. No study-specific consent form was obtained as it was a retrospective analysis. All patients who underwent treatment had a valid consent form which included permission for use of data and information for research.

\section{Chemotherapy protocol}

The chemotherapy regimen consisted of gemcitabine $1,000 \mathrm{mg} / \mathrm{m}^{2}$ and oxaliplatin $100 \mathrm{mg} / \mathrm{m}^{2}$ administered every 2 weeks for a total of 6 cycles. Blood investigations including complete blood count, renal function tests, and liver function tests were obtained prior to each cycle. Chemotherapy was deferred for a week if total leucocyte count was $<3,000$ cells $/ \mathrm{mm}^{3}$ absolute neutrophil count was $<1,500$ cells $/ \mathrm{mm}^{3}$ or platelet count $<100,000$ cells $/ \mathrm{mm}^{3}$. Dose reduction was considered if patients developed more than 2 delays or grade 3 neutropenia or thrombocytopenia or febrile neutropenia.

\section{Radiotherapy protocol}

A planning computed tomography (CT) scan with intravenous contrast with a slice thickness of $3 \mathrm{~mm}$ was acquired through the region of interest with the patient in a supine position using T-bar. The target volume for postoperative radiotherapy included the tumour bed and regional lymph nodes. The clinical target volume (CTV) tumour bed consists of gallbladder fossa and surgical clips with a 1-cm isotropic expansion. Porta hepatis, coeliac, and para-aortic nodes were contoured as regional lymph nodes. Porta

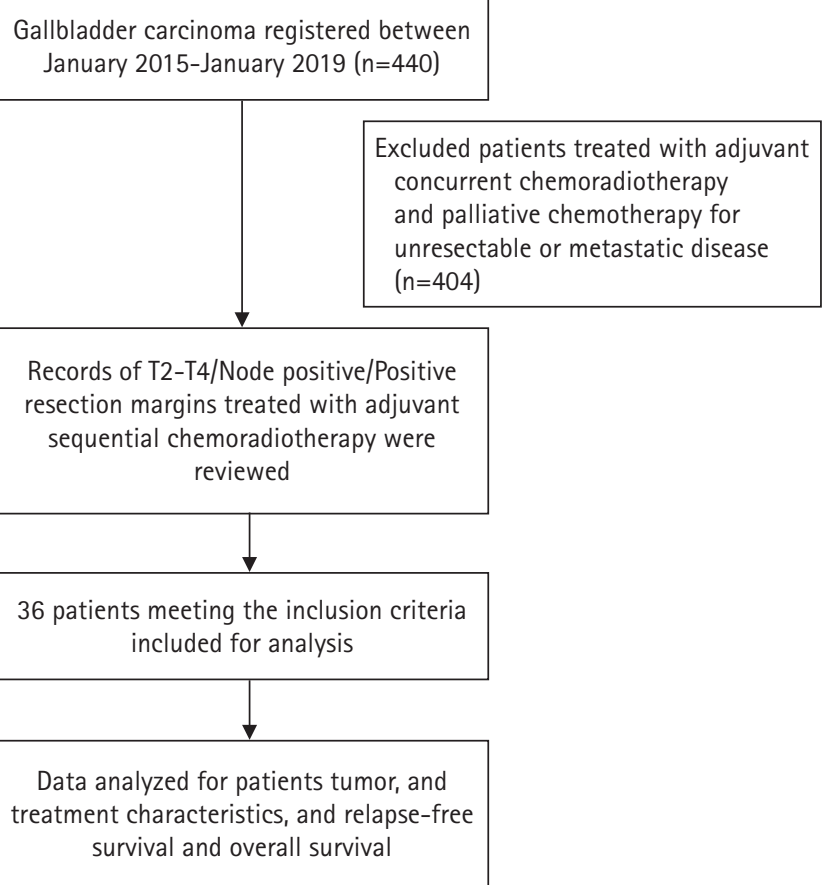

Fig. 1. Flow diagram depicting the selection criteria of the study. 
hepatis was delineated from bifurcation of portal vein to superior mesenteric vein or splenic vein with a 1-cm expansion. The coeliac axis encompasses the proximal $1.5 \mathrm{~cm}$ of the celiac artery with a $1-\mathrm{cm}$ expansion. The aorta is contoured from the superior most contour of either celiac axis or the portal vein, whichever is more superior up to the lower border of the L2 lumbar vertebra. An asymmetric expansion is then given from the aorta, $3 \mathrm{~cm}$ to the right, $1 \mathrm{~cm}$ to the left, $2.5 \mathrm{~cm}$ to the front, and $0.2 \mathrm{~cm}$ to the back to create the para-aortic nodal volume. All the nodal volumes are fused to generate the nodal CTV. A margin of 1-2 cm in the supero-inferior direction and $0.5-1 \mathrm{~cm}$ laterally are given from the CTV to generate the planning target volume (PTV) to account for setup and respiration related uncertainties. The organs-at-risk (OARs) that were contoured include liver, bilateral kidneys, and spinal cord. Radiation therapy was planned by three-dimensional conformal radiation therapy technique (3D-CRT) using three fields and delivered to a total dose of $45 \mathrm{~Gy}$ in 25 fractions at $1.8 \mathrm{~Gy}$ per fraction over 5 weeks. The dose distribution of a patient is shown in Fig. 2.

\section{Follow-up}

After completing adjuvant treatment, follow-up was done once in 3 months for the first 2 years, then every 6 months for the next 3 years, and then annually. The follow-up evaluation comprised of clinical examination, blood investigations including CA19-9, and imaging with ultrasonography and CT as required.

\section{Outcomes assessed}

The primary objective of the study was to estimate the overall survival and relapse-free survival with sequential chemoradiation. The secondary objectives were to assess the relapse pattern, toxicity and factors influencing overall survival and relapse-free survival.

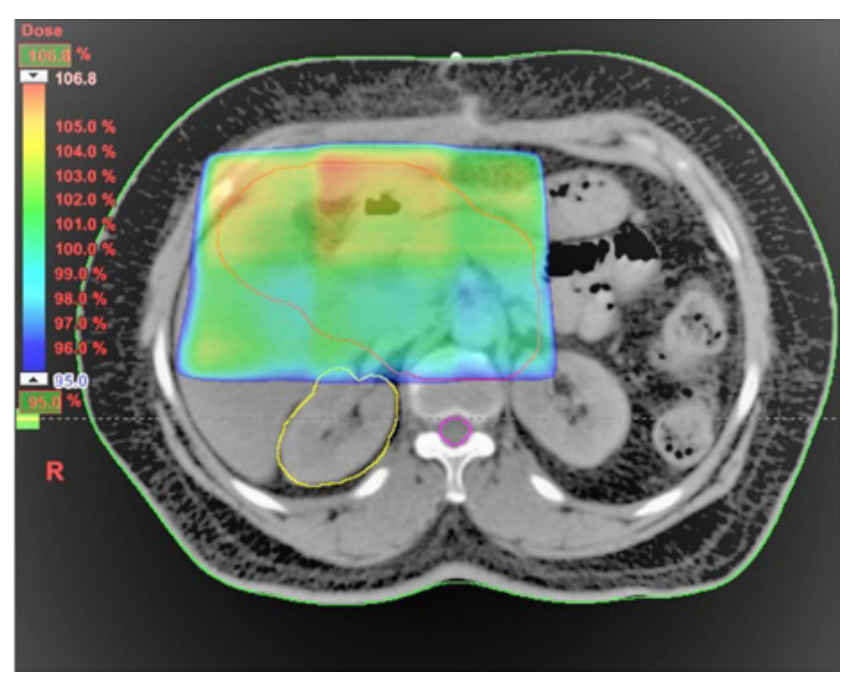

Fig. 2. Dose distribution.
Overall survival and relapse-free survival were calculated from the date of surgery to date of death for any reason or to date of last follow-up and date of relapse, respectively. Relapses were documented as locoregional (recurrence in the tumour bed or regional lymph nodes), distant (recurrence in distant organs or non-regional lymph nodes), or both.

\section{Statistical analysis}

Categorical data were presented as numbers and percentages. Median and range were used to express the numerical data. Overall survival and relapse-free survival were estimated by Kaplan-Meier survival method with 95\% confidence interval (CI). The cut-off date for censoring of patients who were alive was April 2020 and survival status were assessed telephonically if patients were unable to come for follow-up. A univariate analysis using log-rank test and multivariate analysis using Cox proportional hazards model were performed to evaluate the prognostic factors having a significant impact on overall survival. A p-value $<0.05$ was considered statistically significant.

\section{Results}

The study included 36 patients of gallbladder cancers who underwent curative resection and completed adjuvant treatment with sequential chemoradiotherapy. The characteristics of the patients and the disease were summarised in Table 1. The median age was 55.5 years. Approximately two-thirds (61.1\%) of the cases were females and one-fourth (25\%) had incidentally detected carcinomas diagnosed during surgery for gallstone disease. Extended cholecystectomy alone was performed in $86.1 \%$ of patients. Wedge resection of liver, anatomical resection of segments $4 b$ and 5 , and right hepatectomy were performed in 26 (72.2\%), 6 (16.6\%), and 4 (11.1\%) patients, respectively. Additional procedures like sleeve duodenectomy, pancreatoduodenectomy, and resection of colon were performed in 5 patients (13.8\%). Adenocarcinoma and squamous cell carcinoma histology were present in $88.9 \%$ and $8.3 \%$ of patients, respectively. Distribution of patients in various grades of tumour differentiation included: well-differentiated (19.4\%), moderately differentiated (72.2\%), and poorly differentiated tumours (8.3\%). The majority of patients were stage IIIA (41.7\%) and IIIB (25\%). Positive resection margins, lymphovascular space invasion, and perineural invasion were observed in 5 (13.9\%), 7 (19.4\%), and $13(36.1 \%)$ patients, respectively. Approximately 50\% of patients received radiation therapy after completion of chemotherapy.

In general, the treatment was well tolerated and compliance with the protocol was good. A total of 32 patients (88.8\%) completed no less than four cycles of planned chemotherapy; 25 pa- 
Table 1. Patient and disease characteristics

\begin{tabular}{|c|c|}
\hline Characteristic & Value \\
\hline Age (yr) & $55.5(24-76)$ \\
\hline \multicolumn{2}{|l|}{ Sex } \\
\hline Male & $14(38.9)$ \\
\hline Female & $22(61.1)$ \\
\hline \multicolumn{2}{|l|}{ Incidental detection } \\
\hline Yes & $9(25.0)$ \\
\hline No & $27(75.0$ \\
\hline \multicolumn{2}{|l|}{ Type of surgery } \\
\hline \multicolumn{2}{|l|}{ Extended cholecystectomy } \\
\hline Wedge liver resection & $26(72.2)$ \\
\hline Anatomical liver resection & $6(16.6)$ \\
\hline Right hepatectomy & $4(11.1)$ \\
\hline \multicolumn{2}{|l|}{ Additional procedures } \\
\hline Sleeve duodenectomy & $2(5.5)$ \\
\hline Pancreatoduodenectomy & $1(2.7)$ \\
\hline Resection of colon & $2(5.5)$ \\
\hline \multicolumn{2}{|l|}{ Histology } \\
\hline Adenocarcinoma & 32 (88.9) \\
\hline Adenosquamous & $1(2.8)$ \\
\hline Squamous cell carcinoma & $3(8.3)$ \\
\hline \multicolumn{2}{|l|}{ Differentiation } \\
\hline Well differentiated & $7(19.4)$ \\
\hline Moderately differentiated & $26(72.2)$ \\
\hline Poorly differentiated & $3(8.3)$ \\
\hline \multicolumn{2}{|l|}{ T stage } \\
\hline $\mathrm{T} 2 \mathrm{~b}$ & $13(36.1)$ \\
\hline T3 & $23(63.9)$ \\
\hline \multicolumn{2}{|l|}{$\mathrm{N}$ stage } \\
\hline NO & $25(69.4)$ \\
\hline $\mathrm{N} 1$ & $10(27.8)$ \\
\hline N2 & $1(2.8)$ \\
\hline \multicolumn{2}{|l|}{ Stage grouping } \\
\hline IIB & $11(30.6)$ \\
\hline IIIA & $15(41.7)$ \\
\hline IIIB & $9(25.0)$ \\
\hline IVB & $1(2.8)$ \\
\hline \multicolumn{2}{|l|}{ Resection status } \\
\hline Ro & $31(86.1)$ \\
\hline $\mathrm{R} 1$ & 5 (13.9) \\
\hline \multicolumn{2}{|l|}{ Lymphovascular space invasion } \\
\hline Negative & $29(80.6)$ \\
\hline Positive & 7 (19.4) \\
\hline \multicolumn{2}{|l|}{ Perineural invasion } \\
\hline Negative & $23(63.9)$ \\
\hline Positive & $13(36.1)$ \\
\hline
\end{tabular}

Values are presented as median (range) or number (\%). tients (69.4\%) received all six cycles. The causes for cessation of chemotherapy included disease relapse in 5, haematological toxicity in 2, and unknown reasons in 4. Sixteen patients (34.7\%) had haematological toxicity. Grades 1, 2, and 3 leucopoenia were observed in 4 (11.1), 4 (11.1\%), and 1 patient (2.8\%), respectively. Grades 1, 2, 3, and 4 thrombocytopenia were present in 3 (8.3\%), 2 (5.6\%), 1 (2.8\%), and $1(2.8 \%)$ patient, respectively. All patients completed the radiation therapy without any unscheduled interruption. The most common toxicities during radiation were grade 1 skin toxicity and grade 1 vomiting. None of the patients developed radiation therapy related grade 3 and grade 4 complications.

The median follow-up was 21 months (range, 5 to 62 months). The median overall survival and relapse-free survival were 26 months (95\% Cl, 21.4-30.5) and 21 months (95\% Cl, 11.8-30.1), respectively (Fig. 3A, 3B). The 2-year overall survival was 55.1\% (95\% Cl, 37.9\%- 72.3\%) and the 2-year relapse-free survival was $44.7 \%$ (95\% Cl, 27.5\%-61.9\%). Of the 36 patients, recurrences were observed in 19 (52.8\%) as shown in Table 2. Fourteen patients $(73.6 \%)$ had isolated systemic relapses. Both locoregional and distant recurrences were seen in 3 patients (15.7\%); 2 patients $(10.5 \%)$ had isolated locoregional recurrence. Locoregional recurrences were primarily seen as periportal lymph nodes (21\%). The most common sites of distant metastases included liver (36.8\%) and peritoneum (36.8\%). Fig. 4 summarises the various sites of relapses. Of the 19 relapses, 8 (6 distant, 2 combined) occurred in the chemotherapy followed by radiation group and 11 (2 local, 8 distant, 1 combined) in the sandwich radiation group. Eight patients received palliative chemotherapy with capecitabine for relapses, whereas 11 patients were kept under best supportive care in view of poor performance status and disseminated disease. One patient received palliative radiotherapy to a dose of 20 Gy in 5 fractions for the abdominal wall deposit.

A univariate analysis to identify factors influencing relapse free survival and overall survival after radical surgery was carried out. Table 3 summarises the results of the univariate analysis. The findings revealed that nodal status and overall stage grouping, were significantly associated with overall survival $(p<0.05)$. Grading of tumours significantly influenced relapse-free survival ( $p=0.007$ ). Primary tumour stage and lymphovascular invasion demonstrated a close trend for statistically significant association with overall survival. The comparison of survival curves based on nodal staging is shown in Fig. 5. Further, there was a trend towards better overall survival with female sex. All other parameters like positive resection margin, perineural invasion, poorly differentiated tumours, and sequencing of adjuvant therapy (chemotherapy $\rightarrow$ radiotherapy$\rightarrow$ chemotherapy) though associated with poor survival, did not show statistical significance. Table 4 depicts the results of multi- 
(A)

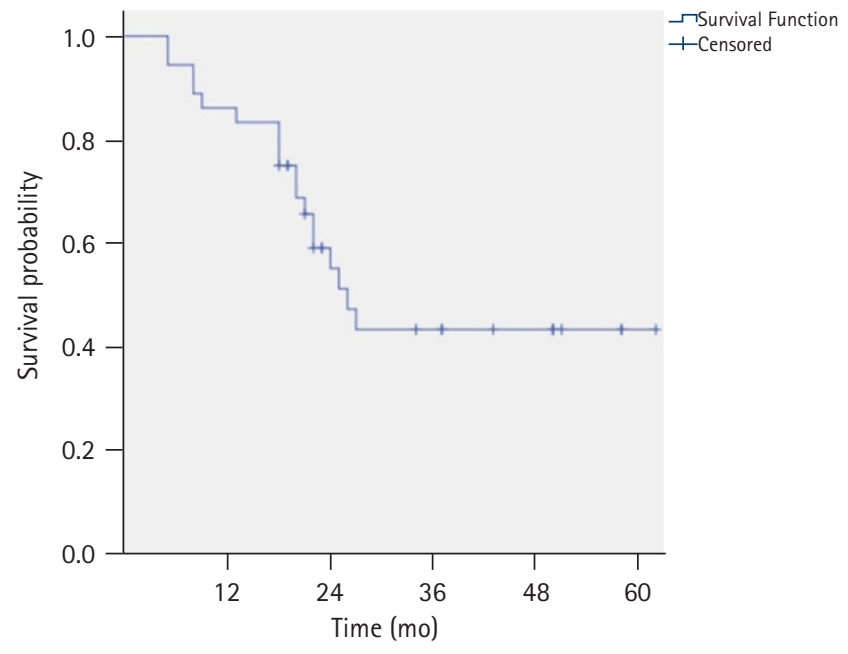

\begin{tabular}{|l|r|r|r|r|r|r|}
\hline $\begin{array}{l}\text { Time in } \\
\text { months }\end{array}$ & 0 & 12 & 24 & 36 & 48 & 60 \\
\hline $\begin{array}{l}\text { Number } \\
\text { at risk }\end{array}$ & 36 & 31 & 14 & 10 & 7 & 1 \\
\hline
\end{tabular}

\section{B}

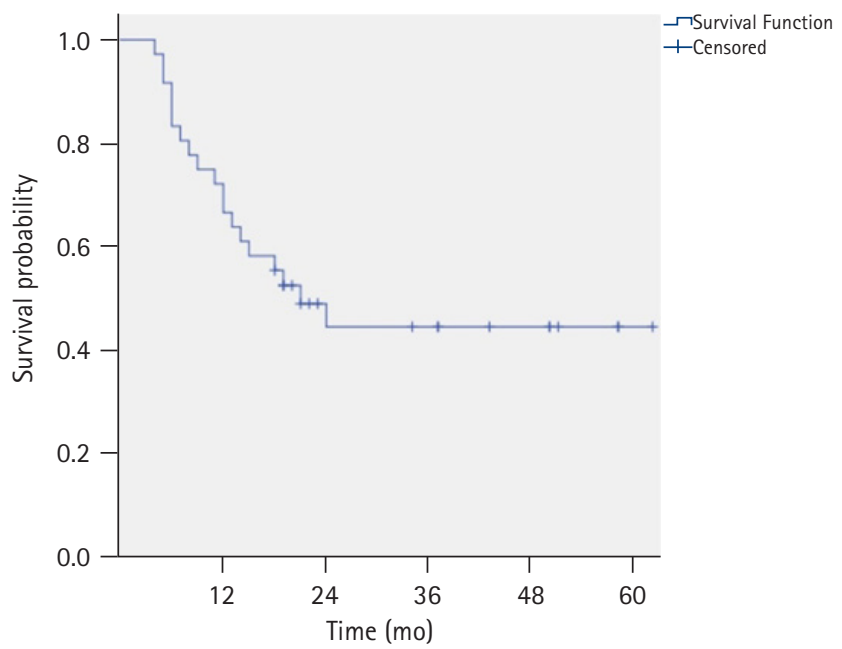

\begin{tabular}{|l|r|r|r|r|r|r|}
\hline $\begin{array}{l}\text { Time in } \\
\text { months }\end{array}$ & 0 & 12 & 24 & 36 & 48 & 60 \\
\hline $\begin{array}{l}\text { Number } \\
\text { at risk }\end{array}$ & 36 & 25 & 10 & 9 & 6 & 1 \\
\hline
\end{tabular}

Fig. 3. The estimated survival probability: (A) overall survival and (B) relapse-free survival.

Table 2. Distribution and pattern of recurrence

\begin{tabular}{lc}
\hline Recurrence & Value \\
\hline No recurrence & $17(47.2)$ \\
Locoregional & $2(5.5)$ \\
Distant & $14(38.8)$ \\
Locoregional + distant & $3(8.3)$ \\
\hline
\end{tabular}

Values are presented as number (\%).

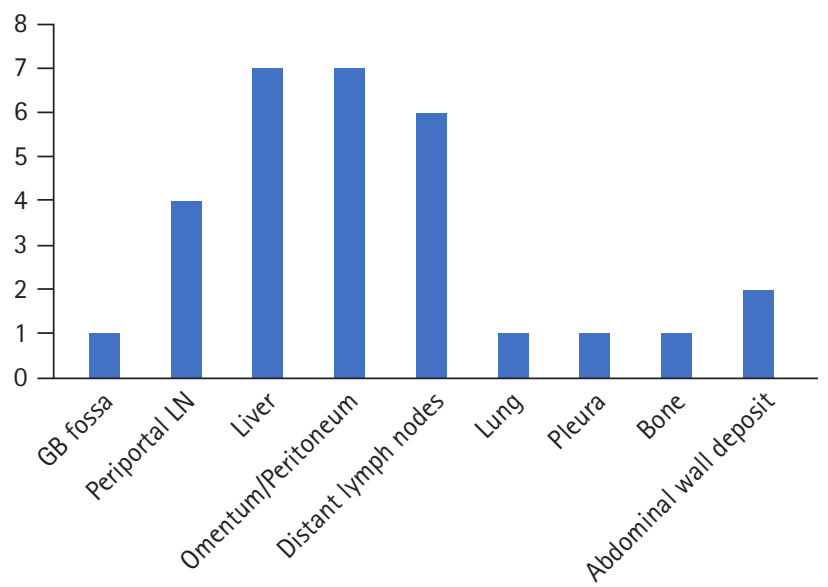

Fig. 4. Sites of relapse. GB, gallbladder; LN, lymph node.

variate analysis for survival performed using Cox proportional hazards model. None of the adverse features including grading, resection status, lymphovascular and perineural invasion have been found to be significantly associated with relapse-free survival and overall survival with sequential chemoradiotherapy.

\section{Discussion and Conclusion}

Carcinomas of gallbladder constitute majority of biliary tract neoplasms and it is more common in females than males [1-4]. In our study, nearly two-thirds of cases were females. As most of these patients often present with either unresectable or metastatic disease, the prognosis is dismal. Among patients who underwent curative resection, the outcomes are often poor owing to locoregional and distant recurrence, justifying the need for adjuvant therapy. Though several adjuvant treatments have been tried, yet a standard consensus adjuvant approach has not been reached, mainly due to lack of robust prospective evidence [10-16]. Though studies utilizing concurrent chemoradiotherapy with either 5-fluorouracil or gemcitabine $[11,13,14]$ are largely available, as such, studies addressing sequential chemoradiation are very limited.

The results of our study suggest that sequential chemoradiation may be effective and better tolerated in patients requiring adjuvant treatment after curative resection and not fit enough to tolerate concurrent chemoradiotherapy. Wang et al. [17] reported a median survival of 8 months $(95 \% \mathrm{Cl}, 8-9)$ with observation in patients with T2 and node positive disease postsurgical resection. However, with adjuvant radiotherapy, authors reported median survival of 15 months (95\% Cl, 13-16) and the 2-year overall survival as 30\%. A 
Table 3. Univariate analysis of RFS and OS using log-rank test

\begin{tabular}{|c|c|c|c|c|c|c|}
\hline Factor & 2-yr RFS (\%) & Median RFS (mo) & $p$-value & $2-y r$ OS $(\%)$ & Median OS (mo) & $p$-value \\
\hline \multicolumn{7}{|l|}{ Sex } \\
\hline Male & 40 & 15 & 0.493 & 25.7 & 20 & 0.087 \\
\hline Female & 48.1 & NR & & 53.7 & NR & \\
\hline \multicolumn{7}{|l|}{ Age (yr) } \\
\hline$\leq 60$ & 41.7 & 19 & 0.518 & 40 & 24 & 0.584 \\
\hline$>60$ & 50.9 & NR & & 48.5 & 26 & \\
\hline \multicolumn{7}{|l|}{ Grade } \\
\hline Well/moderately differentiated & 48.7 & 24 & 0.007 & 45 & 26 & 0.183 \\
\hline Poorly differentiated & 0 & 8 & & 33.3 & 18 & \\
\hline \multicolumn{7}{|l|}{ T stage } \\
\hline $\mathrm{T} 2 \mathrm{~b}$ & 57.7 & NR & 0.170 & 64.5 & NR & 0.099 \\
\hline T3 & 37.3 & 15 & & 31.1 & 22 & \\
\hline \multicolumn{7}{|l|}{$\mathrm{N}$ stage } \\
\hline No & 47 & 21 & 0.202 & 47.8 & 27 & 0.025 \\
\hline $\mathrm{N} 1$ & 40 & 24 & & 35 & 26 & \\
\hline N2 & 0 & 7 & & 0 & 8 & \\
\hline \multicolumn{7}{|l|}{ Stage } \\
\hline IIB & 63.6 & NR & 0.219 & 71.6 & NR & 0.019 \\
\hline IIIA & 37.3 & 15 & & 33.9 & 24 & \\
\hline IIIB & 27.8 & 24 & & 22.2 & 25 & \\
\hline IVB & 0 & 7 & & 0 & 8 & \\
\hline \multicolumn{7}{|l|}{ Resection status } \\
\hline Ro & 45.7 & 24 & 0.449 & 44.8 & 26 & 0.180 \\
\hline $\mathrm{R} 1$ & 40 & 14 & & 40 & 18 & \\
\hline \multicolumn{7}{|l|}{ Lymphovascular space invasion } \\
\hline Negative & 54.2 & 14 & 0.077 & 50.1 & NR & 0.062 \\
\hline Positive & 0 & 21 & & 0 & 20 & \\
\hline \multicolumn{7}{|l|}{ Perineural invasion } \\
\hline Negative & 54.1 & 18 & 0.320 & 47.8 & NR & 0.994 \\
\hline Positive & 28.8 & 21 & & 33.8 & 26 & \\
\hline \multicolumn{7}{|l|}{ Sequence of treatment } \\
\hline Chemotherapy followed by radiotherapy & 50 & 24 & 0.259 & 44.6 & 27 & 0.570 \\
\hline Chemotherapy $\rightarrow$ radiotherapy $\rightarrow$ chemotherapy & 38.9 & 15 & & 41.3 & 22 & \\
\hline
\end{tabular}

RFS, relapse-free survival; OS, overall survival; NR, not reached.

retrospective study on analysis of efficacy of postoperative adjuvant radiotherapy on 127 gallbladder patients by Yang et al. [12] reported a median survival of 16.9 months for stage III patients. Studies with concurrent chemoradiotherapy with fluorouracil showed a median survival of 1.9 years [11]. Results of the present study with sequential chemoradiation show a median survival of 26 months (95\% Cl, 21.4-30.5) and 2-year survival of 55.1\% (95\% $\mathrm{Cl}, 37.9 \%-72.3 \%$ ) which may be non-inferior to concurrent chemoradiotherapy. A median survival of 35 months and 2-year survival rate of $65 \%\left(95 \% \mathrm{Cl}_{1} 53 \%-74 \%\right)$ was reported in the SWOG trial in which cases of cholangiocarcinomas and gallbladder carcinomas were clubbed and received adjuvant chemotherapy fol- lowed by capecitabine based concurrent chemoradiotherapy. Results of our study have lower median survival when compared to adjuvant chemotherapy followed by concurrent chemoradiotherapy, which could be attributed to the lack of concurrent chemotherapy with radiation. However, it must be remembered that in SWOG trial, approximately $52 \%$ of patients experienced grade 3 and $11 \%$ experienced 4 adverse events. The most frequently encountered toxicities were neutropenia, hand-foot syndrome, diarrhoea, and Iymphopenia [15] whereas in the present study of sequential chemoradiation, only two cases experienced grades 3 and 4 haematological side effects. This suggests that sequential chemo radiation may be an effective suitable option in patients who are not 
fit enough to tolerate concurrent chemotherapy.

When we look at the recurrence rates, the published retrospective studies of radiotherapy or chemoradiotherapy in the adjuvant setting have shown a recurrence rate of 39\% to 69\% in the locoregional area and systemic recurrence as high as $80 \%$ whereas our data with sequential chemoradiation exhibited a locoregional recurrence rate of $26.3 \%$ and systemic relapse rate of $38.8 \%$, similar to that reported in SWOG trial in which concurrent capecitabine based chemoradiotherapy was used [8-16,18]. Several factors like nodal status, $T$ stage, resection margin, lymphovascular invasion, perineural invasion, and grade have been shown to influence outcome in several series $[13,16]$. The present study also revealed

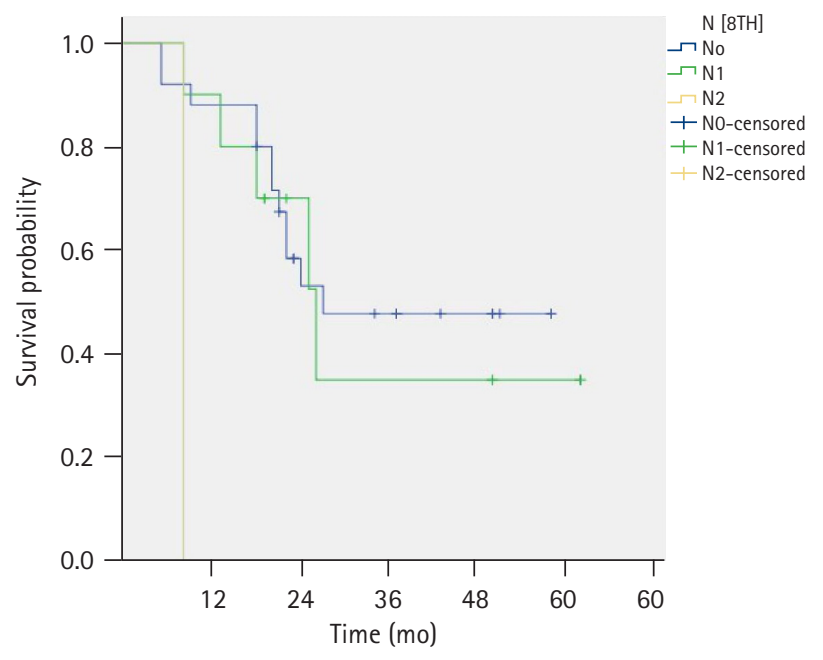

Fig. 5. Comparison of survival curve based on nodal staging. grading to influence relapse-free survival and node positivity, and overall stage grouping as statistically significant factors influencing overall survival on univariate analysis; there was a trend for statistical significance with primary tumour stage and lymphovascular invasion. However, resection margins in our study did not have a statistically significant impact on survival which may be due to small number of patients with positive margins in our study.

Recently, there had been three prospective randomized trials that compared adjuvant chemotherapy versus observation [19-21]. The PRODIGE and BCAT trials demonstrated that adjuvant gemcitabine-based chemotherapy alone was not superior to observation in biliary tract cancers. BILCAP trial, the largest randomized trial to date, though did not meet its primary endpoint showed a significant overall survival benefit with adjuvant capecitabine according to per-protocol analysis. However, in that trial, it was observed that the overall survival was considerably higher in the observation arm also (60\%).

Based on the BILCAP trial, capecitabine is considered as the new standard after surgery and had been incorporated in the American Society of Clinical Oncology 2019 guideline. However, all these trials do not have radiation therapy in the adjuvant arms, thus, making the contribution of postoperative radiotherapy unclear [22].

In conclusion, the present study has revealed that sequential chemoradiation is a feasible, safe and tolerable regimen with acceptable efficacy in the adjuvant setting in patients with adverse features who are not fit enough to tolerate concurrent chemoradiation. The drawback of our study is retrospective design and no comparative arm. In the present situation where adjuvant capecit-

Table 4. Multivariate analysis of RFS and OS using Cox proportional hazards model

\begin{tabular}{|c|c|c|c|c|}
\hline \multirow{2}{*}{ Factor } & \multicolumn{2}{|c|}{ RFS } & \multicolumn{2}{|c|}{ OS } \\
\hline & $\mathrm{HR}(95 \% \mathrm{Cl})$ & $p$-value & $\mathrm{HR}(95 \% \mathrm{Cl})$ & $p$-value \\
\hline \multicolumn{5}{|l|}{ Grade } \\
\hline Well/moderately differentiated & 1 & & 1 & \\
\hline Poorly differentiated & $4.340(0.681-27.642)$ & 0.120 & $1.678(0.246-11.427)$ & 0.597 \\
\hline \multicolumn{5}{|l|}{ N stage } \\
\hline Node negative & 1 & & 1 & \\
\hline Node positive & $1.107(0.399-3.075)$ & 0.845 & $1.278(0.437-3.738)$ & 0.654 \\
\hline \multicolumn{5}{|l|}{ Resection status } \\
\hline Ro & 1 & & 1 & \\
\hline R1 & $1.484(0.342-6.433)$ & 0.598 & $2.011(0.503-8.030)$ & 0.323 \\
\hline \multicolumn{5}{|l|}{ Lymphovascular space invasion } \\
\hline Negative & 1 & & 1 & \\
\hline Positive & $1.098(0.221-5.464)$ & 0.909 & $1.828(0.399-8.374)$ & 0.437 \\
\hline \multicolumn{5}{|l|}{ Perineural invasion } \\
\hline Negative & 1 & & 1 & \\
\hline Positive & $1.383(0.525-3.643)$ & 0.512 & $0.890(0.330-2.404)$ & 0.819 \\
\hline
\end{tabular}

RFS, relapse-free survival; OS, overall survival; $\mathrm{Cl}$, confidence interval. 
abine could be considered as a new standard of care based on the BILCAP trial, the benefit of radiation therapy is unclear. Therefore, prospective randomized controlled trials incorporating adjuvant radiotherapy with chemotherapy are required to confirm the benefit of radiation therapy after curative surgery.

\section{Conflict of Interest}

No potential conflict of interest relevant to this article was reported.

\section{References}

1. Ahn DH, Bekaii-Saab T. Biliary cancer: intrahepatic cholangiocarcinoma vs. extrahepatic cholangiocarcinoma vs. gallbladder cancers: classification and therapeutic implications. J Gastrointest Oncol 2017;8:293-301.

2. The Global Cancer Observatory. Population fact sheet of India [Internet]. Paris, France: International Agency for Research on Cancer, World Health Organization; 2020 [cited 2020 Dec 15]. Available from: https://gco.iarc.fr/today/data/factsheets/populations/356-india-fact-sheets.pdf.

3. Rawla P, Sunkara T, Thandra KC, Barsouk A. Epidemiology of gallbladder cancer. Clin Exp Hepatol 2019;5:93-102.

4. Lazcano-Ponce EC, Miquel JF, Munoz N, et al. Epidemiology and molecular pathology of gallbladder cancer. CA Cancer J Clin 2001;51:349-64.

5. Boerma EJ. Towards an oncological resection of gall bladder cancer. Eur J Surg Oncol 1994;20:537-44.

6. Matsumoto Y, Fujii H, Aoyama H, Yamamoto M, Sugahara K, Suda K. Surgical treatment of primary carcinoma of the gallbladder based on the histologic analysis of 48 surgical specimens. Am J Surg 1992;163:239-45.

7. Gibby DG, Hanks JB, Wanebo HJ, et al. Bile duct carcinoma: diagnosis and treatment. Ann Surg 1985;202:139-44.

8. Kopelson G, Galdabini J, Warshaw AL, Gunderson LL. Patterns of failure after curative surgery for extra-hepatic biliary tract carcinoma: implications for adjuvant therapy. Int J Radiat Oncol Biol Phys 1981;7:413-7.

9. Jarnagin WR, Ruo L, Little SA, et al. Patterns of initial disease recurrence after resection of gallbladder carcinoma and hilar cholangiocarcinoma: implications for adjuvant therapeutic strategies. Cancer 2003;98:1689-700.

10. Bosset JF, Mantion G, Gillet M, et al. Primary carcinoma of the gallbladder: adjuvant postoperative external irradiation. Cancer
1989;64:1843-7.

11. Czito BG, Hurwitz HI, Clough RW, et al. Adjuvant external-beam radiotherapy with concurrent chemotherapy after resection of primary gallbladder carcinoma: a 23-year experience. Int J Radiat Oncol Biol Phys 2005;62:1030-4.

12. Yang $L$, Feng FL, Zhou HH, Sun YJ, Meng Y. [Analysis of the efficacy of postoperative radiotherapy in gallbladder cancer]. Zhonghua Zhong Liu Za Zhi 2013;35:534-9.

13. Gold DG, Miller RC, Haddock MG, et al. Adjuvant therapy for gallbladder carcinoma: the Mayo Clinic Experience. Int J Radiat Oncol Biol Phys 2009;75:150-5.

14. Kresl JJ, Schild SE, Henning GT, et al. Adjuvant external beam radiation therapy with concurrent chemotherapy in the management of gallbladder carcinoma. Int J Radiat Oncol Biol Phys 2002;52:167-75.

15. Ben-Josef E, Guthrie KA, El-Khoueiry AB, et al. SWOG S0809: a phase II intergroup trial of adjuvant capecitabine and gemcitabine followed by radiotherapy and concurrent capecitabine in extrahepatic cholangiocarcinoma and gallbladder carcinoma. J Clin Oncol 2015;33:2617-22.

16. Agrawal S, Gupta PK, Rastogi N, et al. Outcomes of adjuvant chemoradiation and predictors of survival after extended cholecystectomy in gall bladder carcinoma: a single institution experience from an endemic region. J Gastrointest Cancer 2015;46:4853.

17. Wang SJ, Fuller CD, Kim JS, Sittig DF, Thomas CR Jr, Ravdin PM. Prediction model for estimating the survival benefit of adjuvant radiotherapy for gallbladder cancer. J Clin Oncol 2008;26:2112-7.

18. Horgan AM, Amir E, Walter T, Knox JJ. Adjuvant therapy in the treatment of biliary tract cancer: a systematic review and meta-analysis. J Clin Oncol 2012;30:1934-40.

19. Edeline J, Benabdelghani M, Bertaut $A$, et al. Gemcitabine and oxaliplatin chemotherapy or surveillance in resected biliary tract cancer (PRODIGE 12-ACCORD 18-UNICANCER GI): a randomized phase III study. J Clin Oncol 2019;37:658-67.

20. Ebata T, Hirano S, Konishi M, et al. Randomized clinical trial of adjuvant gemcitabine chemotherapy versus observation in resected bile duct cancer. Br J Surg 2018;105:192-202.

21. Primrose JN, Fox RP, Palmer DH, et al. Capecitabine compared with observation in resected biliary tract cancer (BILCAP): a randomised, controlled, multicentre, phase 3 study. Lancet Oncol 2019;20:663-73.

22. Shroff RT, Kennedy EB, Bachini M, et al. Adjuvant therapy for resected biliary tract cancer: ASCO Clinical Practice Guideline. J Clin Oncol 2019;37:1015-27. 